\title{
Frequency characteristics and dynamical behaviors of self-modulation in vertical-cavity surface-emitting lasers
}

\author{
Qiansuo Yang ${ }^{\mathrm{a}, \mathrm{b}, *}$, Akihiro Sasoh $^{\mathrm{a}}$ \\ ${ }^{a}$ Shock Wave Research Center, Institute of Fluid Science, Tohoku University, 2-1-1 Katahira, Aoba, Sendai 980-8577, Japan \\ ${ }^{\mathrm{b}}$ Laboratory of High Temperature Gas Dynamics, Institute of Mechanics, Chinese Academy of Sciences, Beijing 100080, China
}

Received 11 September 2002; received in revised form 21 February 2003; accepted 25 February 2003

\begin{abstract}
The frequency characteristics of a VCSEL with a quarter-wave plate (QWP) and an external reflector are investigated with the translation matrix of the vectorial field. Two series of eigenmode with a shift of half the free spectrum range are linearly polarized, respectively, along the neutral axes of QWP. We also numerically explore the polarization self-modulation phenomenon by using a vectorial laser equation and considering the inhomogeneous broadening of the gain medium. If the external cavity is so short that the shift is bigger than the homogeneous broadening, two stable longitudinal modes oscillate, respectively, on the neutral axes of QWP because they consume different carriers. With a long external cavity, the competition of the modes for the common carriers causes the intensity fluctuation of the modes with a period of one round-trip time of the external cavity.
\end{abstract}

(c) 2003 Published by Elsevier Science B.V.

Keywords: Translation matrix; Optical feedback; Modulation; Quarter-wave plate; Polarization; VCSEL; Modal analysis

\section{Introduction}

The light from a vertical-cavity surface-emitting laser (VCSEL) is linearly polarized along one of the two orthogonal crystal directions, and the linearly polarized modes arise from an intracavity birefringence aligned on the crystal axes (defined as $x$ - and $y$-directions) [1]. When an external reflector and a quarterwave plate (QWP), whose optical axes are oriented at $\pm 45^{\circ}$ with respect to $x$-direction, are used to provide an optical feedback, a VCSEL operates in a polarization self-modulation state at a period slightly longer than twofold the external-cavity round-trip time [2-15]. Most of the reported experiments focus on the intensity waveforms and the frequency characteristics on the neutral axes of QWP and the eigenaxes of VCSELs [2-10].

For a long external cavity, if $x$ - (or $y$-) polarization mode oscillates and the other is almost totally suppressed, which corresponds to the approximate square-wave forms of the intensities, and the polarization

\footnotetext{
${ }^{*}$ Corresponding author. Tel.: +81-222-175-267; fax: +81-222-175-284.

E-mail address: qsyang@rainbow.ifs.tohoku.ac.jp (Q. Yang).
} 
switching happens. With a short external cavity, the square-wave output changes into the sinusoidal curve and antiphase between $x$ - and $y$-polarized modes can be found [3-8]. Another important experimental result is the difference of the frequency characteristics on the two eigenaxes of the VCSELs and on the neutral axes of the QWP. The spectrum of $x$ - or $y$-polarized modes usually consists in a few successive modes with a frequency separation, $\Delta v_{ \pm}$, of about half the free spectrum range (FSR) of the external cavity. On each neutral axis of the QWP, however, there is one comb of successive longitudinal modes with a mode separation of $2 \Delta v_{ \pm}$and the frequency shift between the two combs equals $\Delta v_{ \pm}$. They gradually degenerate into two stable longitudinal modes by shortening the external cavity. Meanwhile, on each eigenaxis of the VCSEL, there are two successive modes with a separation of $\Delta v_{ \pm}$, which corresponds to the sinusoidal modulation of the intensities and antiphase effect [7].

Several models are used to explain the dynamical behaviors of the VCSELs [6-15]. By using the model of beatnote between the eigenmodes, Brunel et al. [6] and Ropars et al. [9] successfully explained the comb structure of the laser spectra on the neutral axes of the QWP in VCSELs and He-Ne laser. The beatnote model was also in agreement with Ginovart et al.'s experimental results in which a short external cavity was used [7]. The other models focused on the investigation of the dynamical behaviors such as the waveforms and the rf spectrum of the intensities on the eigenaxes of the VCSEL. In fact, because the transitions with different frequencies from the conduction to the valence bands are produced by different carriers, the inhomogeneous broadening of the gain medium should be considered in the analysis of the dynamical behaviors above [16]. This broadening effect makes the oscillation of different eigenmodes on the neutral axes of the QWP consume different carriers if the external cavity is so short that the shift is bigger than the homogeneous broadening of the carriers. For a shift being smaller than the homogeneous broadening, however, the competition of the eigenmodes for the common carriers induces the periodic fluctuation of the eigenmode intensities.

In this paper, we analyse the frequency characteristics of the eigenmodes of a VCSEL with a QWP and an external reflector in Section 2 by using the translation matrix for the vectorial field. Then, Section 3 presents the establishment of the vectorial laser equation. In order to take into account the inhomogeneous broadening of the gain medium, we divide the carriers into three parts: the first two parts are, respectively, consumed by the two series of eigenmode and the third, as the common carriers, interacts with both of them. In Section 4, the dynamical behaviors and the frequency characteristics are numerically investigated and a brief discussion on the results is also given.

\section{Translation matrix and frequency characteristics}

The typical structure of a polarization self-modulation VCSEL is shown in Fig. 1, where a QWP is inserted into the external cavity oriented at $45^{\circ}$ with respect to the eigenaxes of the VCSEL. On the two

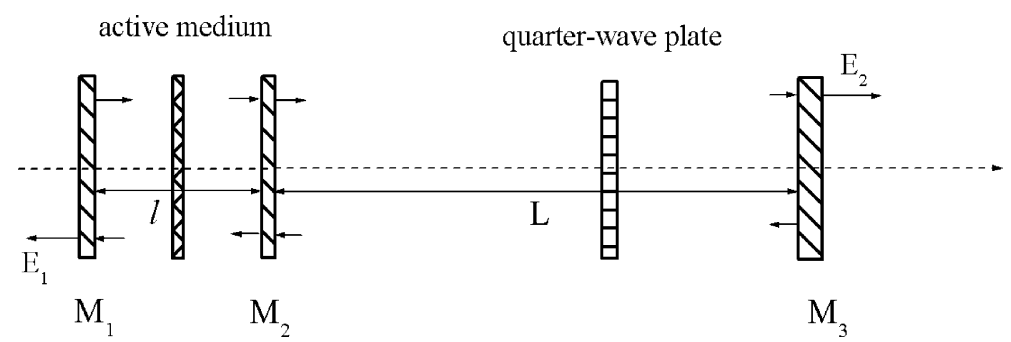

Fig. 1. General scheme of a polarization self-modulation VCSEL with an external cavity and a QWP where the angle of the neutral axes of the QWP with respect to the eigenaxes of the VCSEL is $45^{\circ}$. 
neutral axes of the QWP, the forward and backward running wave components and the outputs at the two sides of the laser can be expressed as

$$
E=\left(\begin{array}{c}
E_{\mathrm{f}+} \\
E_{\mathrm{b}+} \\
E_{\mathrm{f}-} \\
E_{\mathrm{b}-}
\end{array}\right), \quad E_{1}=\left(\begin{array}{c}
0 \\
E_{1+} \\
0 \\
E_{1-}
\end{array}\right) \quad \text { and } \quad E_{2}=\left(\begin{array}{c}
E_{2+} \\
0 \\
E_{2-} \\
0
\end{array}\right)
$$

where the subscripts "f" and " $b$ " denote the forward and the backward propagation of the light fields and "+" and "_" the fast and the slow axes of the QWP, respectively.

The vectorial translation matrixes of the two mirrors of the VCSEL and the external reflector, $T_{1}, T_{2}$ and $T_{3}$, can be written as $(\delta=1,2,3)[17]$ :

$$
T_{\delta}=\left(\begin{array}{cccc}
A_{\delta} & B_{\delta} & 0 & 0 \\
C_{\delta} & D_{\delta} & 0 & 0 \\
0 & 0 & A_{\delta} & B_{\delta} \\
0 & 0 & C_{\delta} & D_{\delta}
\end{array}\right)
$$

where $A_{\delta} D_{\delta}-B_{\delta} C_{\delta}=1$. For simplicity, the elements of the matrixes are assumed to be real. Moreover, in order to keep the electric fields with the same sign before and after being reflected by the mirrors, the elements of the matrixes follow the equations: $A_{\delta}=D_{\delta}=t_{\delta}^{-1}, B_{1}=C_{1}=r_{1} t_{1}^{-1}, B_{2}=C_{2}=-r_{2} t_{2}^{-1}$ and $B_{3}=C_{3}=-r_{3} t_{3}^{-1}$, where $r_{\delta}$ and $t_{\delta}$ are the reflectance and transmittance of the mirrors for the electric field.

The translation matrix of the running wave propagation in the external cavity is expressed as:

$$
T_{\text {EXT }}=\left(\begin{array}{cccc}
\mathrm{e}^{-\mathrm{j} k_{0} L} & 0 & 0 & 0 \\
0 & \mathrm{e}^{\mathrm{j} k_{0} L} & 0 & 0 \\
0 & 0 & \mathrm{e}^{-\mathrm{j} k_{0} L} & 0 \\
0 & 0 & 0 & \mathrm{e}^{\mathrm{j} k_{0} L}
\end{array}\right),
$$

where $L$ and $k_{0}$ are the external-cavity length and the corresponding wavenumber, respectively. However, by considering the birefringence effect inside the VCSEL, the translation matrix changes into

$$
T_{\mathrm{V}}=\frac{1}{2}\left(\begin{array}{cccc}
\mathrm{e}^{-\mathrm{j} k_{x} l}+\mathrm{e}^{-\mathrm{j} k_{y} l} & 0 & \mathrm{e}^{-\mathrm{j} k_{x} l}-\mathrm{e}^{-\mathrm{j} k_{y} l} & 0 \\
0 & \mathrm{e}^{\mathrm{j} k_{x} l}+\mathrm{e}^{\mathrm{j} k_{y} l} & 0 & \mathrm{e}^{\mathrm{j} k_{x} l}-\mathrm{e}^{\mathrm{j} k_{y} l} \\
\mathrm{e}^{-\mathrm{j} k_{x} l}-\mathrm{e}^{-\mathrm{j} k_{y} l} & 0 & \mathrm{e}^{-\mathrm{j} k_{x} l}+\mathrm{e}^{-\mathrm{j} k_{y} l} & 0 \\
0 & \mathrm{e}^{\mathrm{j} k_{x} l}-\mathrm{e}^{\mathrm{j} k_{y} l} & 0 & \mathrm{e}^{\mathrm{j} k_{x} l}+\mathrm{e}^{\mathrm{j} k_{y} l}
\end{array}\right),
$$

where $k_{x}$ and $k_{y}$ are the wavenumbers on $x$ - and $y$-directions, respectively, and $l$ is the cavity length of the VCSEL. On the other hand, the electric fields are amplified by the gain medium when they pass through it. Therefore, the translation matrix for the amplification can be written as

$$
T_{\mathrm{g}}=\left(\begin{array}{cccc}
A_{\mathrm{g}+} & 0 & 0 & 0 \\
0 & D_{\mathrm{g}+} & 0 & 0 \\
0 & 0 & A_{\mathrm{g}-} & 0 \\
0 & 0 & 0 & D_{\mathrm{g}-}
\end{array}\right)
$$

where $A_{\mathrm{g}+}, A_{\mathrm{g}-}, D_{\mathrm{g}+}^{-1}$ and $D_{\mathrm{g}-}^{-1}$ are the static amplification coefficients of the forward and backward running waves, respectively. When the electric fields pass through the QWP, a phase delay of $\pi / 2$ is added to the components on the slow axis and the corresponding translation matrix is defined as:

$$
T_{\mathrm{QWP}}=\left(\begin{array}{cccc}
1 & 0 & 0 & 0 \\
0 & 1 & 0 & 0 \\
0 & 0 & \mathrm{e}^{-\mathrm{j} \pi / 2} & 0 \\
0 & 0 & 0 & \mathrm{e}^{\mathrm{j} \pi / 2}
\end{array}\right) .
$$


Since the translation matrix can be used to describe the propagation of the electric fields in the cavity, the steady state outputs at the two sides of the laser satisfy the following matrix equation:

$$
E_{2}=T_{3} T_{\mathrm{EXT}} T_{\mathrm{QWP}} T_{2} T_{\mathrm{V}} T_{\mathrm{g}} T_{1} E_{1} .
$$

Specifically, we introduce the variables $\Omega_{+}, \Omega_{-}, \sigma_{+}$and $\sigma_{-}$, which are defined by

$$
\begin{aligned}
& \Omega_{+}=\mathrm{e}^{\mathrm{j} k_{x} l}+\mathrm{e}^{\mathrm{j} k_{y} l}, \quad \Omega_{-}=\mathrm{e}^{-\mathrm{j} k_{x} l}+\mathrm{e}^{-\mathrm{j} k_{y} l}, \\
& \sigma_{+}=\mathrm{e}^{\mathrm{j} k_{x} l}-\mathrm{e}^{\mathrm{j} k_{y} l}, \quad \sigma_{-}=\mathrm{e}^{-\mathrm{j} k_{x} l}-\mathrm{e}^{-\mathrm{j} k_{y} l} .
\end{aligned}
$$

Considering the cavity of the VCSELs to be very short and $k_{x} \approx k_{y}$, we can get

$$
\left|\Omega_{ \pm}\right| \gg\left|\sigma_{ \pm}\right| \text {. }
$$

By introducing Eqs. (1)-(6) into Eq. (7) and using inequality (9), we can obtain that the eigen-wavenumbers of the electric fields on the fast and slow axis of the QWP, $k_{+}$and $k_{-}$, respectively, satisfy

$$
\begin{aligned}
& D_{\mathrm{g}+} \Omega_{+}\left(r_{2} r_{3}+\mathrm{e}^{\mathrm{j} 2 k_{+} L}\right)=A_{\mathrm{g}+} \Omega_{-}\left(r_{1} r_{3}+r_{1} r_{2} \mathrm{e}^{\mathrm{j} 2 k_{+} L}\right), \\
& D_{\mathrm{g}-} \Omega_{+}\left(r_{2} r_{3}+\mathrm{e}^{\mathrm{j} 2 k_{-} L+\mathrm{j} \pi}\right)=A_{\mathrm{g}-} \Omega_{-}\left(r_{1} r_{3}+r_{1} r_{2} \mathrm{e}^{\mathrm{j} 2 k_{-} L+\mathrm{j} \pi}\right) .
\end{aligned}
$$

From Eqs. (10a) and (10b), it is straightforward that the frequency shift between the two series of eigenmodes is $c / 4 L$ and the mode separation of each series of eigenmodes is $c / 2 L$, respectively, which are the same as the theoretical results derived by Brunel and Ropars et al. and also in agreement with the experiments $[6,7,9]$. For a typical VCSEL, there is the relation: $k_{x} l \approx n \pi \approx k_{y} l$, where $n$ is a small integer, and, therefore, we can get the following solutions of Eqs. (10a) and (10b):

$$
\begin{aligned}
& A_{\mathrm{g}+} D_{\mathrm{g}+}^{-1}=\frac{1+r_{2} r_{3}}{r_{1}\left(r_{2}+r_{3}\right)}, \quad 2 k_{+} L=2 n \pi, \\
& A_{\mathrm{g}-} D_{\mathrm{g}-}^{-1}=\frac{1+r_{2} r_{3}}{r_{1}\left(r_{2}+r_{3}\right)}, \quad 2 k_{-} L=2 n \pi+\pi, \\
& A_{\mathrm{g}+} D_{\mathrm{g}+}^{-1}=\frac{1-r_{2} r_{3}}{r_{1}\left(r_{2}-r_{3}\right)}, \quad 2 k_{+} L=2 n \pi+\pi, \\
& A_{\mathrm{g}-} D_{\mathrm{g}-}^{-1}=\frac{1-r_{2} r_{3}}{r_{1}\left(r_{2}-r_{3}\right)}, \quad 2 k_{-} L=2 n \pi .
\end{aligned}
$$

By comparing them with those for the corresponding solitary laser, one can see that the thresholds of the stable modes in Eqs. (11a) and (11b) are lower and those of the unstable 'antimodes' in Eqs. (12a) and (12b) are higher [15].

\section{Model for numerical simulation}

Considering the optical feedback and the rotation of the polarization by the QWP, we modify the vectorial laser equation proposed in [18] to investigate laser dynamical behaviors. Due to the isotropical gain of VCSELs, the vectorial laser equation can be written as:

$$
\frac{\mathrm{d} E_{x}}{\mathrm{~d} t}=\frac{k}{\pi} \int_{0}^{2 \pi}\left[E_{x} \cos \theta+E_{y} \sin \theta\right] \cos \theta \cdot Z \mathrm{~d} \theta-k E_{x}+\beta E_{y}\left(t-\tau_{0}\right),
$$




$$
\begin{aligned}
& \frac{\mathrm{d} E_{y}}{\mathrm{~d} t}=\frac{k}{\pi} \int_{0}^{2 \pi}\left[E_{x} \cos \theta+E_{y} \sin \theta\right] \sin \theta \cdot Z \mathrm{~d} \theta-k E_{y}+\beta E_{x}\left(t-\tau_{0}\right), \\
& \frac{\mathrm{d} Z}{\mathrm{~d} t}=P-\frac{Z}{\tau_{\delta}}-\frac{2 k}{\pi}\left|E_{x} \cos \theta+E_{y} \sin \theta\right|^{2} Z,
\end{aligned}
$$

where $E_{x}$ and $E_{y}$ are the electric fields on the eigenaxes of the VCSEL. $k$ is the decay rate of the electric field in the cavity and $\tau_{\delta}$ the lifetime of the carrier. $P$ and $Z$ are the injection current and the angular density of the carrier and $\tau_{0}$ and $\beta$ are one round-trip time and the feedback strength of the external cavity, respectively.

Viewing the electric field on the neutral axes of the QWP, Eqs. (13a)-(13c) can be changed into the following forms:

$$
\begin{aligned}
& \frac{\mathrm{d} E_{+}}{\mathrm{d} t}=\frac{k}{\pi} \int_{0}^{2 \pi}\left[E_{+} \cos \vartheta+E_{-} \sin \vartheta\right] \cos \vartheta \cdot Z \mathrm{~d} \vartheta-k E_{+}+\beta E_{+}\left(t-\tau_{0}\right), \\
& \frac{\mathrm{d} E_{-}}{\mathrm{d} t}=\frac{k}{\pi} \int_{0}^{2 \pi}\left[E_{+} \cos \vartheta+E_{-} \sin \vartheta\right] \sin \vartheta \cdot Z \mathrm{~d} \vartheta-k E_{-}-\beta E_{-}\left(t-\tau_{0}\right), \\
& \frac{\mathrm{d} Z}{\mathrm{~d} t}=P-\frac{Z}{\tau_{\delta}}-\frac{2 k}{\pi}\left|E_{+} \cos \vartheta+E_{-} \sin \vartheta\right|^{2} Z,
\end{aligned}
$$

where $E_{+}$and $E_{-}$are the electric fields on the neutral axes of the QWP, satisfy the following relationships: $E_{+}=\left(E_{x}+E_{y}\right) / \sqrt{2}, E_{-}=\left(E_{x}-E_{y}\right) / \sqrt{2}$. The definition of $\vartheta=\theta+45^{\circ}$ is also used.

From the analysis in Section 2, we know that $E_{+}$and $E_{-}$are the two series of eigenmodes with a shift of $c / 4 L$. Considering the inhomogeneous broadening effect of the gain medium, the carriers are divided into three parts: the first two parts amplify $E_{+}$and $E_{-}$, respectively, and the third interacts with both of them, as shown in Fig. 2. For a short external cavity, the frequency shift $c / 4 L$ is so big that the oscillations of $E_{+}$and $E_{-}$are mainly supported by different carriers, as shown in Fig. 2(a). However, the longer the external cavity, the smaller the frequency shift between $E_{+}$and $E_{-}$, and the stronger competition of them for the common carriers, as shown in Fig. 2(b). Therefore, Eqs. (14a)-(14c) becomes

$$
\begin{aligned}
\frac{\mathrm{d} E_{+}}{\mathrm{d} t} & =\frac{k}{\pi} \int_{0}^{2 \pi}\left\{E_{+} \cos ^{2} \vartheta \cdot\left(Z_{1}+Z_{3}\right)+E_{-} \sin \vartheta \cos \vartheta \cdot Z_{3}\right\} \mathrm{d} \vartheta-k E_{+}+\beta E_{+}\left(t-\tau_{0}\right), \\
\frac{\mathrm{d} E_{-}}{\mathrm{d} t} & =\frac{k}{\pi} \int_{0}^{2 \pi}\left\{E_{-} \sin ^{2} \vartheta \cdot\left(Z_{2}+Z_{3}\right)+E_{+} \cos \vartheta \sin \vartheta \cdot Z_{3}\right\} \mathrm{d} \vartheta-k E_{-}-\beta E_{-}\left(t-\tau_{0}\right), \\
\frac{\mathrm{d} Z_{1}}{\mathrm{~d} t} & =P_{1}-\frac{Z_{1}}{\tau_{\delta}}-\frac{2 k}{\pi}\left|E_{+} \cos \vartheta\right|^{2} Z_{1}, \\
\frac{\mathrm{d} Z_{2}}{\mathrm{~d} t} & =P_{2}-\frac{Z_{2}}{\tau_{\delta}}-\frac{2 k}{\pi}\left|E_{-} \sin \vartheta\right|^{2} Z_{2}, \\
\frac{\mathrm{d} Z_{3}}{\mathrm{~d} t} & =P_{3}-\frac{Z_{3}}{\tau_{\delta}}-\frac{2 k}{\pi}\left|E_{+} \cos \vartheta+E_{-} \sin \vartheta\right|^{2} Z_{3},
\end{aligned}
$$




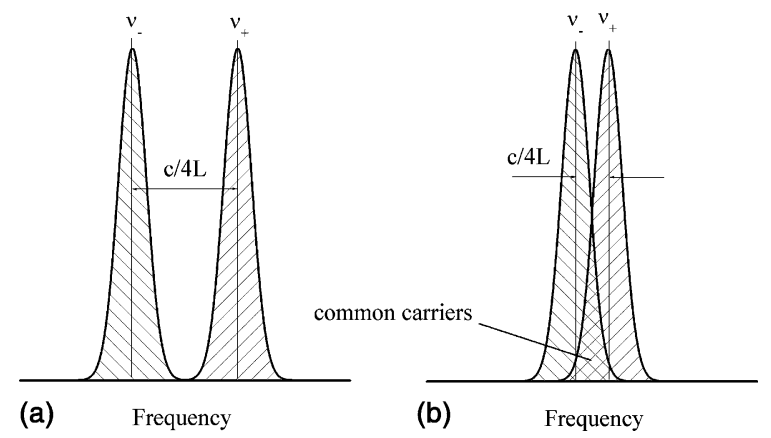

Fig. 2. The carrier distribution profiles for the two eigenmodes $E_{+}$and $E_{-}$for (a) a short external cavity and (b) a long external cavity.

where $Z_{1}, Z_{2}$ and $Z_{3}$ are the angular distribution density of the three parts of carrier and $P_{1}, P_{2}$ and $P_{3}$ the corresponding injection currents, respectively.

From the analysis in Section 2, one can find that the energy exchange between the two series of eigenmodes on the neutral axes of the QWP is described in Eq. (4) after they pass through the birefringence medium. For simplicity, we have neglected this effect because of the following reasons. First, from Eqs. (4) and (8a) and (8b), we can conclude that the exchange is very weak. On the other hand, for the two components on the neutral axes of the QWP with the same frequency, one is a normal longitudinal mode and the other is an anti-mode. Thus, the latter is suppressed by the former because of their different thresholds, as described by Eqs. (11a), (11b) and (12a), (12b).

\section{Results and discussion}

Using Eqs. (15a)-(15e), we numerically study the frequency characteristics and the dynamical behaviors of polarization self-modulation of the VCSELs. In the following simulations, we simulate the two typical cases: a short external cavity and a long one.

\subsection{A short external cavity}

When the external cavity becomes so short and the frequency shift $c / 4 L$ between $E_{+}$and $E_{-}$so big that their interaction through the consumption of the common carriers does not exist, which means $P_{3}=0$ and $Z_{3}=0$, Eqs. (15a)-(15e) can be simplified into:

$$
\begin{aligned}
& \frac{\mathrm{d} E_{+}}{\mathrm{d} t}=\frac{k}{\pi} \int_{0}^{2 \pi} E_{+} \cos ^{2} \vartheta \cdot Z_{1} \mathrm{~d} \vartheta-k E_{+}+\beta E_{+}\left(t-\tau_{0}\right), \\
& \frac{\mathrm{d} E_{-}}{\mathrm{d} t}=\frac{k}{\pi} \int_{0}^{2 \pi} E_{-} \sin ^{2} \vartheta \cdot Z_{2} \mathrm{~d} \vartheta-k E_{-}-\beta E_{-}\left(t-\tau_{0}\right), \\
& \frac{\mathrm{d} Z_{1}}{\mathrm{~d} t}=P_{1}-\frac{Z_{1}}{\tau_{\delta}}-\frac{2 k}{\pi}\left|E_{+} \cos \vartheta\right|^{2} Z_{1}, \\
& \frac{\mathrm{d} Z_{2}}{\mathrm{~d} t}=P_{2}-\frac{Z_{2}}{\tau_{\delta}}-\frac{2 k}{\pi}\left|E_{-} \sin \vartheta\right|^{2} Z_{2} .
\end{aligned}
$$




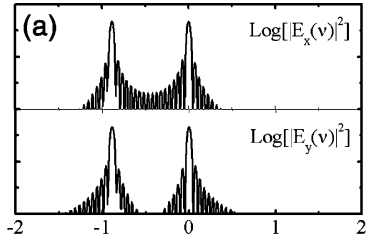

Frequency (unit:c/4L)

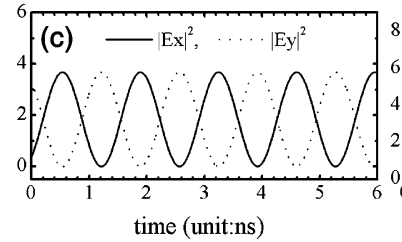

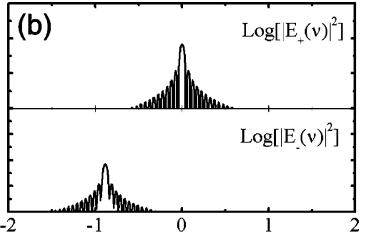

Frequency (unit:c/4L)

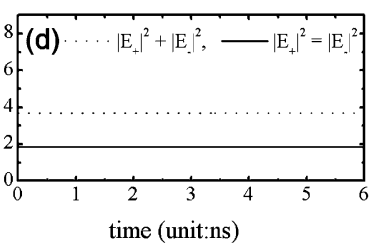

Fig. 3. (a, b) The spectra on the eigenaxes of the VCSEL and the neutral axes of the QWP, (c, d) the intensity waveforms on the eigenaxes of the VCSEL and on the neutral axes of the QWP where $k=0.5 \mathrm{ps}^{-1}, \tau_{\delta}=1 \mathrm{~ns}, P_{1}=P_{2}=1.45, P_{3}=0, \beta=0.008$ and $L=9 \mathrm{~cm}$.

In the numerical analysis of the laser equations, we take the same parameter values for gain medium as in other papers [10,14,15]: $k=0.5 \mathrm{ps}^{-1}, \tau_{\delta}=1 \mathrm{~ns}, P_{1}=P_{2}=1.45, \beta=0.008$ and $L=9 \mathrm{~cm}$. Meanwhile, the photon lifetime is 1 ps and the threshold of the injection current 1 . Fig. 3 shows the frequency spectra and the intensity waveforms on the neutral axes of the QWP and the eigenaxes of the VCSEL. There is a singlefrequency electric field on each neutral axis of QWP and the system operates at a CW state, as shown in Fig. 3(b) and (d). On the eigenaxes of the VCSEL in Fig. 3(a) and (c), the intensity beats and the spectra of the two modes above can be seen and the corresponding mode separation, $\Delta v_{ \pm}$, are slight lower than $c / 4 L$ because of the amplification of the light by the gain medium. From Eq. (16b), we can see that the frequency shift of $E_{-}$with respect to $E_{+}$leads to the change of the sign in the front of $\beta$ and, therefore, the two modes are all stable normal modes.

\subsection{A long external cavity}

With a long external cavity, the interaction of the two modes due to the amplification of the modes by the common carriers cannot be neglected. This coupling effect causes the periodic fluctuation of the ei-
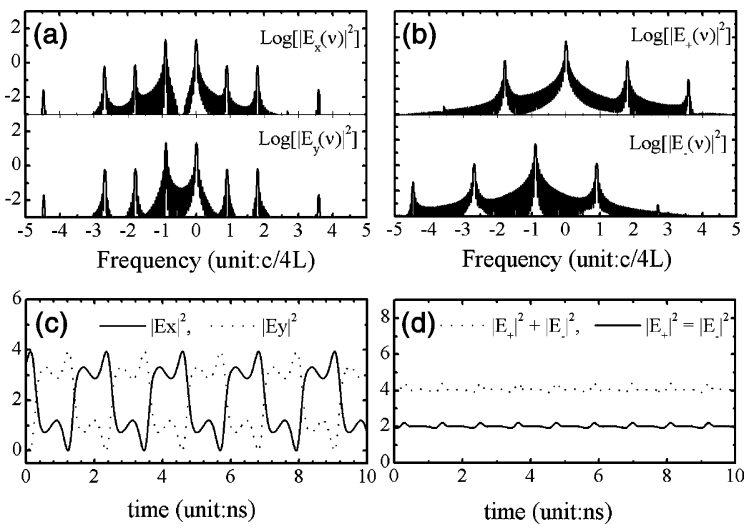

Fig. 4. Same as Fig. 3 but for $P_{1}=P_{2}=1.4, P_{3}=0.05, \beta=0.008$ and $L=15 \mathrm{~cm}$. 
genmode intensities. Fig. 4 shows the intensity waveforms and the spectra when the external cavity and the pumps are takes as $L=15 \mathrm{~cm}, P_{1}=P_{2}=1.45$ and $P_{3}=0.05$, respectively. As analyzed in Section 2, the two series of eigenmodes, $E_{+}$and $E_{-}$, have a frequency shift $\Delta v_{ \pm}$and consist in a few successive longitudinal modes with a separation of $2 \Delta v_{ \pm}$, as shown in Fig. 4(b), which are the same as the previous experiment results [6,9]. On each one of the eigenaxes of VCSEL, the spectrum is a successive longitudinal modes with a mode separation of $\Delta v_{ \pm}$. The competition of $E_{+}$and $E_{-}$for the common carriers also changes the waveforms of the intensities on the eigenaxes of the VCSEL from the sinusoidal modualtion into the similar square-wave, as shown in Fig. 4(c).

To increase $P_{3}$ results in the oscillation of more longitudinal modes on neutral axes of the QWP. However, it is impossible to simulate the intensity waveforms on the eigenaxes of the VCSEL quantitatively because the adiabatic approximation has been used in the vectorial laser equations $[18,19]$. In general, the transverse relaxation rate (TRR) of the gain medium of a VCSEL is very high and it belongs a class B laser. Unfortunately, TRRs for most of gain media are unknown within our knowledge [19]. For a polarization self-modulation VCSEL, there should be a stronge dependence of the intensity waveforms on TRR due to a very short cavity and a not long external cavity. It is obvious that lower TRR results in fewer longitudinal modes because TRR is directly proportional to the polarization rate of the gain medium by the eletcric field.

\section{Conclusion}

In summary, by considering the structure of a VCSEL with a QWP and an external reflector and using translation matrix for a vectorial field, we obtain the mode separation and the frequency shift of the two eigenmodes on the neutral axes of the QWP. Incorporating the inhomogeneous broadening, we provide a set of laser equation to simulate the polarization self-modulation phenomenon. For a short external cavity, there is no interaction between $E_{+}$and $E_{-}$because they consume different carriers and possess a frequency shift of $\Delta v_{ \pm}$slightly lower than $c / 4 L$. With a long external cavity, however, the competition of $E_{+}$and $E_{-}$ for the common carriers causes their fluctuation with a period of about one round-trip time, which corresponds to a few successive longitudinal modes on each neutral axis of the QWP, with a separation of $2 \Delta v_{ \pm}$.

\section{Acknowledgements}

The authors acknowledge helpful discussions with Dr. Hua Li and Dr. G. Jagadeesh.

\section{References}

[1] K.D. Choquette, D.A. Richie, R.E. Leibenguth, Appl. Phys. Lett. 64 (16) (1994) 2062.

[2] N. Badr, I.H. White, M.R.T. Tan, Y.M. Houng, S.Y. Wang, Electron. Lett. 30 (15) (1994) 1227.

[3] W.H. Loh, A.T. Schremer, C.L. Tang, IEEE Photon. Technol. Lett. 2 (7) (1990) 467.

[4] W.H. Loh, Y. Ozeki, C.L. Tang, Appl. Phys. Lett. 56 (26) (1990) 2613.

[5] S. Jiang, Z. Pan, M. Dagenais, R.A. Morgan, K. Kojima, Appl. Phys. Lett. 63 (26) (1993) 3545.

[6] M. Brunel, M. Vallet, G. Ropars, A. Le Floch, F. Bretenaker, G. Joulie', J.-C. Keromnes, Phys. Rev. A 55 (2) (1997) 1391.

[7] F. Ginovart, F. Robert, P. Besnard, J. Opt. B: Quantum Semiclass. Opt. 1 (1999) 646.

[8] F. Robert, P. Besnard, M.L. Chares, G.M. Stephan, IEEE J. Quantum Electron. 33 (1997) 2231.

[9] G. Ropars, P. Langot, M. Brunel, M. Vallet, F. Bretenaker, A. Le Floch, K.D. Choquette, Appl. Phys. Lett. 70 (20) (1997) 2661.

[10] H. Li, A. Hohl, A. Gavrielides, H. Hou, K.D. Choquette, Appl. Phys. Lett. 72 (19) (1998) 2355. 
[11] C.L. Tang, Quantum Semiclass. Opt. 10 (1998) R51.

[12] W.H. Loh, C.L. Tang, Opt. Commun. 85 (1991) 283.

[13] W.H. Loh, C.L. Tang, IEEE J. Quantum Electron. 27 (3) (1991) 389.

[14] C. Masoller, N.B. Abraham, Appl. Phys. Lett. 74 (8) (1999) 1078.

[15] M. Sciamanna, T. Erneux, F. Rogister, O. Deparis, P. Mégret, M. Blondel, Phys. Rev. A 65 (4) (2002) 041801.

[16] M. San Miguel, Q. Feng, J.V. Moloney, Phys. Rev. A. 52 (2) (1995) 1728.

[17] P. Yeh, A. Yariv, C.-S. Hong, J. Opt. Soc. Am. 67 (1977) 423.

[18] Q.S. Yang, P.Y. Wang, J.H. Dai, H.J. Zhang, IEEE J. Quantum Electron. 34 (7) (1998) 1135.

[19] C.O. Weiss, R. Vilaseca, in: Dynamics of Lasers, VCH, Weinheim, 1991, p. 81. 